\title{
A Machine Learning Approach for Modular Workflow Performance Prediction
}

\author{
Alok Singh \\ San Diego Supercomputer Center \\ University of California San Diego \\ La Jolla, CA, USA \\ a1singh@ucsd.edu
}

\author{
Arvind Rao \\ San Diego Supercomputer Center \\ University of California San Diego \\ La Jolla, CA, USA \\ a3rao@ucsd.edu \\ Ilkay Altintas \\ San Diego Supercomputer Center \\ University of California San Diego \\ La Jolla, CA, USA \\ altintas@ucsd.edu
}

\author{
Shweta Purawat \\ San Diego Supercomputer Center \\ University of California San Diego \\ La Jolla, CA, USA \\ shpurawat@ucsd.edu
}

\begin{abstract}
Scientific workflows provide an opportunity for declarative computational experiment design in an intuitive and efficient way. A distributed workflow is typically executed on a variety of resources and it uses a variety of computational algorithms or tools to achieve the desired outcomes. Such a variety imposes additional complexity in scheduling these workflows on large scale computers. As computation becomes more distributed, insights into expected workload that a workflow presents become critical for effective resource allocation. In this paper, we present a modular framework that leverages Machine Learning for creating precise performance predictions of a workflow. The central idea is to partition a workflow in such a way that makes the task of forecasting each atomic unit manageable and gives us a way to combine the individual predictions efficiently. We recognize a combination of an executable and a specific physical resource as a single module. This gives us a handle to characterize workload and machine power as a single unit of prediction. The modular approach of the presented framework allows it to adapt to highly complex nested workflows and scale to new scenarios. We present performance estimation results of independent workflow modules executed on the XSEDE SDSC Comet cluster using various Machine Learning algorithms. The results provide insights into the behavior and effectiveness of different algorithms in the context of scientific workflow performance prediction.
\end{abstract}

\section{CCS CONCEPTS}

- Computing methodologies Machine learning • Computing methodologies $\sim$ Artificial intelligence • Information systems $\sim$ Information systems applications

Permission to make digital or hard copies of all or part of this work for personal or classroom use is granted without fee provided that copies are not made or distributed for profit or commercial advantage and that copies bear this notice and the full citation on the first page. Copyrights for components of this work owned by others than ACM must be honored. Abstracting with credit is permitted. To copy otherwise, or republish, to post on servers or to redistribute to lists, requires prior specific permission and/or a fee. Request permissions from Permissions@acm.org.

WORKS'17, November 12-17, 2017, Denver, CO, USA

(c) 2017 Association for Computing Machinery.

ACM ISBN 978-1-4503-5129-4/17/11\$15.00

DOI: https://doi.org/10.1145/3150994.3150998

\section{KEYWORDS}

Machine Learning, Scientific Workflows, Performance Prediction, Parallel computing, Distributed computing, Exascale computing

\section{ACM Reference format:}

A. Singh, A. Rao, S. Purawat, and I. Altintas. 2017. A Machine Learning Approach for Modular Workflow Performance Prediction. In Proceedings of 12th Workshop on Workflows in Support of Large-Scale Science, Denver, CO, USA, November 2017 (WORKS'17), 11 pages.

DOI: $10.1145 / 3150994.3150998$

\section{INTRODUCTION}

Computing resource utilization and efficient processing of tasks using scheduling algorithms are evolving areas of research. Dynamic scheduling algorithms which utilize estimates of the workload presented by a particular task perform better when the estimates are accurate. Improvements in accuracy of forecasts can positively impact scheduling performance [1]. This intuitively makes sense, since it is a vital piece of information, and has definite say in ascertaining the amount of resources (including time) a given task consumes. In this paper, we build on this intuition to present a modular resource centric performance prediction framework that forecasts a workflow's performance footprint.

Scientific workflows are means to declaratively define and execute what needs to be accomplished in computational and data science studies. Among their many advantages [2] is the flexibility of implementation of execution choices across many platforms as long the task at hand is completed in expected manner. Scientific workflow schedulers aim to allow the investigation of multiple possible paths of execution and decide the best route under the given scenario. Specifically, scientific workflows have emerged as highly capable tools to execute complex experiments [3]. Accurate estimates of resource requirements can reduce costs and improve system utilization.

Approach and Contributions. We approach the problem of estimating performance footprint of a large workflow by dividing the task into smaller tasks involving fewer variations, and hence reducing the complexity of non-linear interactions we should capture.

Central to any divide and conquer strategy is the concept of granularity. How fine should each atomic piece be before we stop 
partitioning the problem further? Researchers have tackled the problem of estimation by picking different granularities ranging from finer low-level workload estimation [4] to coarser workflow level predictions [5]. Fine grained evaluation of each instruction does give handle on each step of the workflow, however, it increases the complexity of the prediction task itself. The cost of measurement becomes the bottleneck. On the other end, when the entire workflow is evaluated as a single atomic unit, it leaves significant amount of details such as differential parallelism of each sub task or arbitrary nesting of each sub component, which can degrade the precision of the estimates.

We leverage the idea that combination of a resource and a workload has a significant impact on deciding the set point of performance footprint of a task. We capture this conceptual coupling in a construct we call 'module'. As shown in Fig 1, a module is a combination of an executable and a hardware resource where the executable runs on. We demarcate the boundary of a module by using the circumference of a resource. This coupling allows us to utilize Machine Learning agents that specialize in predicting performance of instruction sets on a given resource architecture. Since the physical resource becomes constant for a given prediction agent, it can dig deeper into capturing the non-linear interaction of workflow task's instructions with the resource, and become more sensitive to variation in performance as the instructions vary.

Once each learning agent generates the predictions, the framework can combine them into a single performance prediction for the workflow by leveraging the structure of the Physical Resource Execution Plan (PREP). A physical resource execution plan is detailed mapping of each abstract task to a physical machine where it will run. The abstract workflow only declares what needs to be accomplished. The PREP gives information about the coupling of various tasks to physical machines. The composition thus results in giving a data-driven prediction for the workflow.

We use a scientific workflow called the Microbiome Taxonomy and Gene Abundance workflow (MTGA) to generate profiling data on the SDSC Comet cluster. Comet is part of XSEDE resources, an NSF funded infrastructure available to researchers. MTGA contains various sub tasks which are fairly independent and represent different tasks. The resource centric agents are tested in such a way, which represents their ability to generalize well on unseen workflows. Hence, even though MTGA is used as a specific example for proof of concept, the prediction ability is tested in a way that captures a model's ability to generalize well on workflow tasks which were not executed before on a particular resource.

Outline. The remainder of this paper is organized as follows: Section II gives an overview of related work. Section III presents our Modular Resource Centric Approach to Performance Prediction. Section IV explains our profiling infrastructure that gathers data for training. Section V delineates our Learning Architecture that performs grid search on a large set of data partitions with different Machine Learning models. Section VI shows the Machine Learning algorithms under consideration in this paper. Section VII presents our representative use case, the MTGA workflow, and Section VIII and IX show results when different volumes of data is presented to learning algorithms. Section X showcases results of recursive feature reduction aimed at reducing the problem size, and Section XI concludes the paper summarizing key highlights and contributions.

\section{RELATED WORK}

The scientific community has tackled the efficient and accurate "Workflow Performance Prediction" problem from multiple angles, involving diverse techniques and platforms. New ways of leveraging developments in Machine Learning to make performance forecasts [6]-[8] was one of the active areas. We present in this section, highlights of works, which have contributed to performance forecasting research using machine learning.

[9] characterizes a workflow using a set of attributes, and uses a distance measure to evaluate the similarity between two workflows. Each attribute can potentially have different weight. The work deploys a local learning framework to make predictions and use induction modeling for making Grid workflow predictions. [10] presents a level based prediction model that utilizes data dependencies to partition a workflow into levels. A performance prediction is made for each level using the workflow structure and job characteristics. It primarily uses workflow characteristics for its prediction.

[11] investigates Machine Learning algorithms for making resource utilization predictions for two bioinformatics applications. It uses both system and application attributes for characterization. It proposes an approach of adding regression functions to leaves of a binary tree classifier, called Predicting Query Runtime. [5] present an architecture for characterizing and modeling of workflow performance. However, the study only makes use of linear models. Since the system does not have prior relations (linear etc.) information, it has to build all possible combinations of model types and blocks.

[12] uses similarity templates to make online workflow execution predictions but has to perform exhaustive search to find templates. [13] present a way to evaluate predictability of an algorithm. The paper proposes a tradeoff between a black box vs. a white box approach in order to generalize well to new algorithms. The approach ignores system features and uses a single idle machine. [14] proposes a black-box method (NIMO) that automatically executes workflows on idle resources to generate training data, and perform cost estimation. The resource workbench which acts as a test bed consists of heterogeneous resources. NIMO, however, only uses multivariate linear regression.

[15] evaluates Support Vector Machines, Neural Networks and Linear Regression in the context of predicting a web application's performance. The experiments use a virtual machine based web server and a database server hosted separately. Support Vectors gives best performance for the web application considered. This may not necessarily be a generalizable fact, when we evaluate Support Vectors for MTGA workflow, presented in current paper. [16] evaluates Artificial Neural Network and Support Vector Machines for predicting performance of virtual machine based applications. The paper exposes a single model's inability to capture nuances in resource utilization and proposes that sub-modeling can shrink estimation errors drastically. [17] uses a cluster of sub-models, each characterizing a sub-portion of a system, to make cross-platform forecasts for Internet services. The approach utilizes expert level knowledge of the inherent fabric of the Internet Services, but does demonstrate that composition of large number of simple models can generate a reliable predictor. [18] proposes a technique specific to FORTRAN but can be applied to other languages. It utilizes abstract operations to characterize workloads, and maps a program to these abstract operations. The overall running time is a linear sum of number of occurrences of each operation multiplied by the time it takes for each operation.

[19] proposes an online estimation technique that links needs of a task to the input data size. The system detects correlations between task needs and input data size. [20] uses exponential smoothing method that works for Grid Environments. The system switches dynamically between multiple changing parameter values depending on the state. The model parameters are taken from optimal values for a previous instance of similar observation. [21] utilizes a black box probabilistic model to estimate workflow estimation makespan in grid infrastructures. It uses a random latency variable to capture the 
overall grid variability. [22] uses multiple prediction schemes and picks the most likely accurate candidate. This selection from an array of models is claimed to be generating near optimal solutions. [23] introduces a time series pattern based forecasting strategy. The approach deals with long and short duration tasks separately.

Our approach tackles the problem of granularity by using resource boundary to demarcate an atomic unit of prediction. We deploy a modular approach to combine each atomic prediction into an overall workflow performance estimate. This dichotomy allows for future improvement to adapt new techniques for atomic predictions, while keeping the composition of individual estimates tied to the overall workflow structure.

\section{MODULAR RESOURCE CENTRIC APPROACH}

At an abstract level, a workflow is a declaration of how the input data should be processed and formatted before presenting the output to a user. Workflows provide an intuitive ability to take leap from algorithmic concepts to practical implementation on physical machines, without losing control of what needs to be accomplished, and yet provide freedom to decide 'how' it needs to be executed. This balance of deterministic outcomes coupled with flexibility to choose your own implementation, is one of the significant advantages of using scientific workflows and is a very important capability for future growth of scientific computing. We anticipate that exascale computing infrastructure of the future will have significant portions of their workloads in form of workflows.

Scheduling jobs on distributed platforms dynamically is a challenge for efficient workflow execution. Every workflow represents a different workload; hence schedulers needs to handle each one differently. We present a modular resource centric prediction framework, that characterizes a workflow and forecasts its performance footprint with respect to the resource it is executed on. This performance prediction can act as critical piece of information for achieving efficient scheduling and optimal utilization of resources.

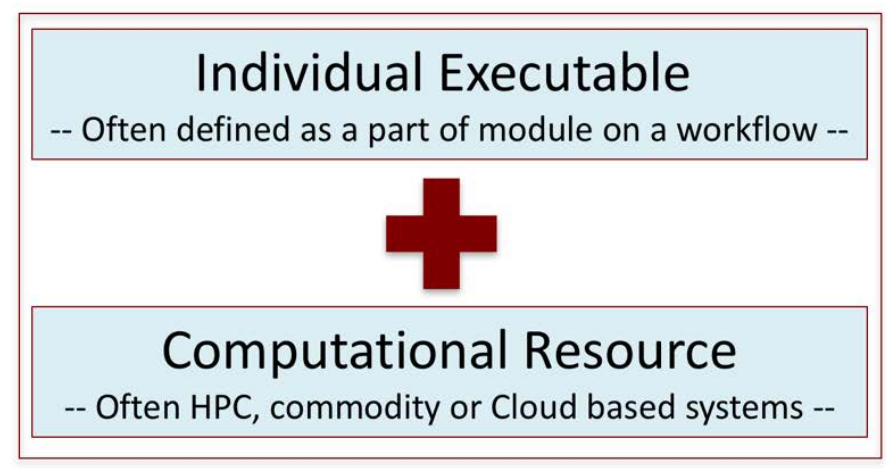

Fig 1 A Module is an atomic unit of prediction representing an executable and the compute node where it runs. This resourceboundary allows reduction in complexity and enables Machine Learning agents to focus on fewer moving parts.

The Modular Resource Centric Prediction Framework (MRCP), shown in Fig 2, leverages Machine Learning algorithms to estimate performance. Each step in abstract workflow is mapped to one or more physical machines, depending on the task, its level of parallelism and nesting. The framework utilizes this physical mapping of executable tasks to physical machines, called Physical Resource Execution Plan (PREP), to delineate a 'module'. A module is a tightly coupled combination of an executable and a physical node on which it runs. A module also represents an atomic single unit of prediction in the MRCP. It allows an algorithm to specialize and learn about behavior of a specific set of instructions on a given hardware configuration. A module represents the natural amalgamation of workload and resource power that the machine brings to the table. The predicted temporal graph shown in Fig 2, has the same structure as the PREP, and each node contains the time required to execute the respective module. The composition algorithm takes the temporal graph and combines individual predictions to generate overall workflow performance prediction. There have been several attempts to perform workflow performance prediction. Some approaches go to fine grained analysis of each line of code, and lose on speed, while others treat the entire workflow as a single prediction task and lose on accuracy. Our modular approach balances this tradeoff between complexity and precision by using the boundaries of a physical machine to demarcate our atomic unit of prediction - a module.

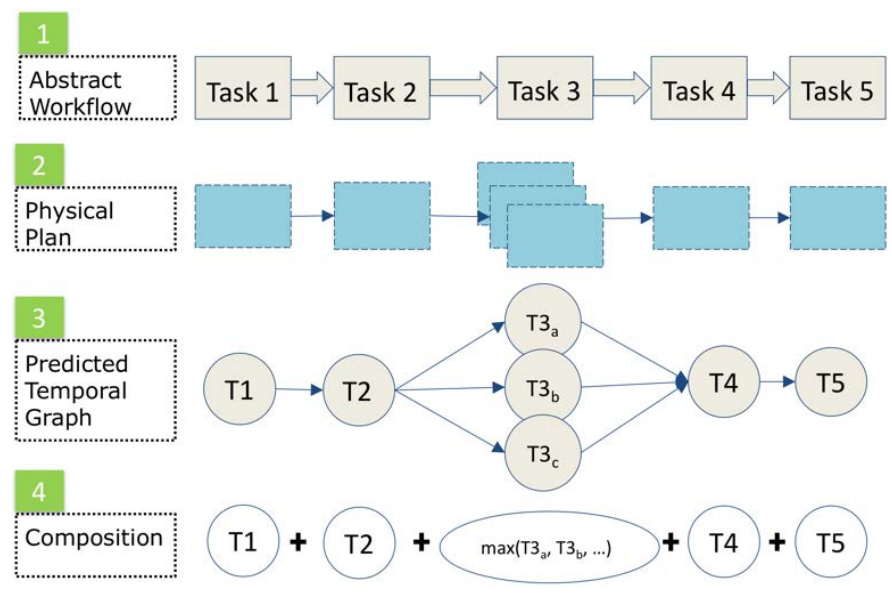

Fig 2 Modular Resource Centric Prediction Framework (MRCP) decouples the prediction algorithm (Step 3) from composition (Step 4), thus, allowing for future improvement by leveraging new techniques for precise prediction of workflow modules.

Our modular approach to prediction inherently adapts to handle nesting of tasks and distributed workflows. The framework uses resource centric learning agents who specialize in predicting performance of a set of instructions on that machine (i.e. resource). It then combines the estimates from each learning agent into a single prediction by utilizing Physical Resource Execution Plan (PREP). The PREP gives insight into the operations used during composition of outputs - such as addition during serial tasks that occur one after the other, or picking the maximum length task in case of parallel execution.

Our modular approach enables us to create specialized Machine Learning agents for predicting performance. This method adapts and scales well as the diversity and nesting of workflow tasks increase. These learning agents can have a microscopically zoomed in view of small variations among different workflow tasks since these agents are tied to specific resource types. This gives each learning agent the freedom to fine tune itself as per the instructions it is presented without the need to understand how resource variation affects the prediction. The framework handles variation of physical machines by having diverse agents, much like a sales team each specializing in sales to a different set of clients.

\section{PROFILING FRAMEWORK}


A big challenge in developing Machine Learning agents is to train them on using data from real computing clusters that executes scientific workflows. In this section, we present a profiling framework that captures behavior of workflow tasks on distributed computing clusters. The design of this framework and the Machine Learning agents is generalizable to new scenarios. The profiling framework automates the task of submitting workflow tasks to compute nodes, and the task of collecting profiling information from these compute nodes with minimal interference to actual performance. The profiling framework is also designed to allow control of resource configuration, should the underlying architecture permit such a mechanism. This framework allows us to capture variation in performance of a set of instructions when the underlying physical hardware resource is changed or when the size of the input to the module is altered.

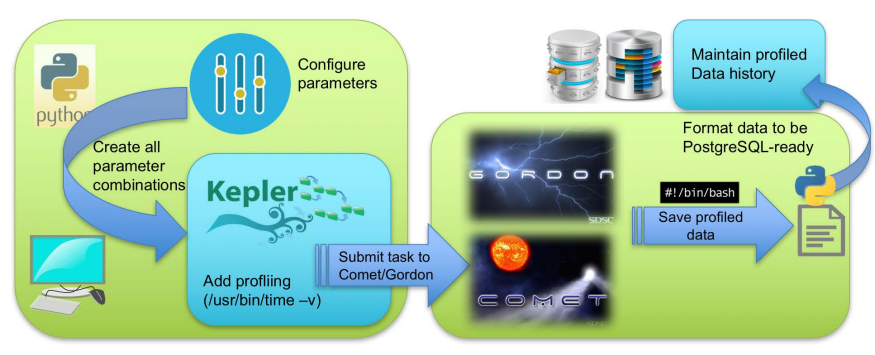

Fig 3 Profiling Framework is designed with scalability at its core. The Kepler workflow system interfaces with the computing resource (such as Comet). A local Python scripts automates the job of generating all possible profiling experiment combinations and invokes Kepler to submit tasks to the distributed infrastructure.

The profiling framework in Fig 3 has two main components - one that runs on local machine, and other that executes on a compute cluster. We utilize Kepler workflow system [24] to interface with the job submission queue of the compute cluster. This gives us two distinct advantage - ability to parametrize the execution and delegate the task of job submission to Kepler workflow system. We utilize a Python program to generate combinations of parameters under which experiments need to be performed and trigger Kepler workflow system to submit jobs for each combination. The job script has parameters for controlling the resource capabilities and for diverting the profiled output to a designated location on cluster for further analysis. The parameters pertaining to workflow itself can also be passed using this script. Once the job completes, profile information is collected from the designated place on the cluster and transformed into SQL friendly format for easy ingestion into analytical tools such as Pandas.

We use numactl tool (Non-Uniform Memory Access) for controlling the memory assignment and varying the number of CPUs. In order to keep the measurements accurate, we needed a tool that was lightweight and did not overload the system. We have used '/usr/bin/time' utility for capturing the system performance. This is an inbuilt utility tied closely to the operating system, and provides enough information for running our analytics experiments.

In the experimental data presented in this paper, we vary the input file size and the number of CPUs available to each task. The total number of runs is proportional to the cross product of number of data partitions (i.e. file sizes), number of CPU variations, and number of tasks in our representative workflow. We keep other hardware (e.g. memory) allocation fixed for all experiments. We plan to extend the range of variation in future by controlling other dimensions such as amount of memory available at a given compute node.

\section{Program Instruction Characterization:}

In order to characterize the workflow of each module, we used the Milepost [25] compiler to extract program instruction information. Milepost extracts 55 control flow graph related features, that distinguish each module based on the workload represented by their program instructions. The features that characterize a module's workflow include information such as number of edges in the control flow graph, number of binary floating-point operations, number of instructions that do pointer arithmetic, sum of number of phi-nodes at the beginning of a basic block, number of conditional branches, number of assignment instructions, number of calls that return a pointer etc. [26] gives a complete list of features. We added two more features: \# of CPUs and Input File Size in GBs. These program features together with resource characteristics compactly represent a module as a feature vector.

\section{LEARNING ARCHITECTURE}

Learning architecture for modular prediction treats each module as a unit of prediction. Given a set of modules and their performance profiles on different resources, the learning architecture aims to learn to predict execution times for unseen modules. The central theme of learning is to be able to generalize to new unseen modules, and not overfit to the training samples. The deviation of predicted values from expected reality is measured through Root Mean Square Error (RMSE).

The objective of the learning architecture is to select the Machine Learning model that can be trained to optimally predict execution times of unseen modules. To ensure that we can find a model's behavior when subjected to lesser amount of data (training samples), we perform incremental learning. In the incremental learning, we partition the profiled data into regions containing less information at the start and gradually increase the information content of the training set. Each training session is independently performed.

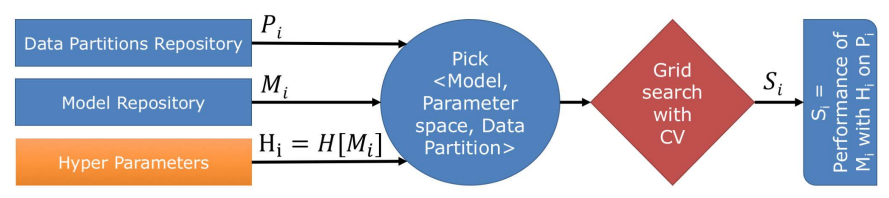

Fig 4 Learning Architecture: Each permutation of partition from data partition repository and model from model repository is picked, and grid search is performed over the respective hyper parameters using cross-validation. The best estimator's performance as measured on left out data is compared for model selection.

shows different parts of our Learning Architecture. The Model Repository contains the candidate algorithms. The Hyper Parameters are customized to each learning algorithm since each one has different parametrization. To maximize the amount of available training data, we leverage a threefold cross validation process. The optimal parameter for a given model is reached by performing exhaustive grid search in coordination with cross validation. This precludes the need for a separate validation set, and increases the amount of information available for training. The last element of variability in the learning architecture controls the data partition size 
that the model is fed during each round of grid search. We start with a small partition (containing only two of four profiled modules) and increase to encapsulate all four modules. This data distillation process gives us an opportunity to capture a model's sensitivity to the amount of training data.

In the following sections, we present results in two different categories: the strict and lenient training. The analysis is performed using Scikit-learn library in Python [27]. The datasets and the code are openly available on GitHub (https://github.com/wordssdsc/IPPD). The entire profiled dataset contains four independent workflow modules. The strict training restricts the model to not look at any sample from a module that is present in the test set. The lenient training allows the model to learn from samples from modules whose samples are also present in the test set. This leaks some information about modules present in the test set (but not the actual samples). In either case, no sample is present in both the test and train data sets, hence every sample in the test set is unseen from model's perspective. Having a separate test set enables us to verify a model's ability on new samples (i.e. generalization on samples it has not seen before), and hence avoid falling into the trap of overfitting.

\section{MACHINE LEARNING ALGORITHMS}

\section{Linear Models:}

We selected Lasso, Ridge and ElasticNet models to be part of model repository. Each of these models utilizes different technique for keeping the model complexity under control. The complexity is measured by the sizes of weights learnt by each model. Lasso uses L1 penalty, Ridge employs L2 penalty and ElasticNet deploys a combination of L1 and L2 regularization. Lasso gives an added advantage of feature selection while learning. The optimization objective for each linear model is shown: (1) Lasso, (2) Ridge, (3) ElasticNet.

$\frac{1}{2 n_{\text {samples }}}|| y-X w||_{2}^{2}+\alpha|| w||_{1}$

$\frac{1}{2 n_{\text {samples }}}|| y-X w||_{2}^{2}+\alpha|| w||_{2}$

$\frac{1}{2 n_{\text {samples }}}|| y-X w||_{2}^{2}+\alpha \rho|| w||_{1}+\frac{\alpha(1-\rho)}{2} \| w||_{2}^{2}$

The hyper-parameter range for $\alpha$ used in Grid Search is presented in the three-number format: $[l, u, n]$ which denotes $n$ points spaced evenly on log scale in range $[l, u]$. Lasso: $\alpha \in$ $\left[10^{-4}, 10^{10}, 50\right]$, Ridge: $\alpha \in\left[10^{-35}, 10^{25}, 100\right]$, and ElasticNet: $\alpha \in\left[10^{-25}, 10^{25}, 40\right], \rho \in[.9, .75, .5, .25, .1]$.

\section{Ensemble Methods:}

We investigate the performance of two ensemble algorithms in the context of workflows: Random Forest [28] and Gradient Boosting [29]. Ensemble methods combine output from a large set of predictors in a way that makes the overall predictor much more accurate than individual forecasts.

Random Forest model uses Out of Bag Errors (OOB). OOB error is the mean prediction error on a sample $\xi_{i}$, using all the trees that do not have $\xi_{i}$ in their training set. Training samples are drawn with replacement from the input data set.

Boosting methods construct many weak predictors, which perform slightly better than random predictors. Gradient Boosting sequentially adds decision trees to reduce residual errors, which are calculated by evaluating gradients. Random Forest on the other hand develops decision trees independently in parallel with focus in reducing variance on each decision node.

The number of estimators are varied from 2000 to 10000 in steps of 2000. The learning rate for Gradient Boosting goes over the following discrete values: [0.001, 0.005, 0.01]. For both models, the depth is not restricted by an upper limit, the minimum number of samples required to split an internal node is two, the minimum number of samples necessary at a leaf node is one. The leaf nodes are not constrained by a minimum weight fraction nor is there an upper limit to the number of leaf nodes. The minimum impurity mandatory for splitting a node is $10^{-7}$.

\section{Support Vector Regression:}

Support Vector Machines (SVM) [30], [31] have proved extremely successful in forecasting, and we have included 4 different kernels in our experiments. The central idea of SVM regression is to first map raw data onto a high dimensional plane using a kernel. This is followed by creating a linear regression model in the higher dimensional space. Each kernel performs a different mapping of the raw dataset, and hence gives different performance. We are interested in finding if a given kernel is more suitable in the context of workflow performance prediction.

The four kernels in SVM that compete against each other are: Linear, Polynomial, Sigmoid, and Radial Basis Function (RBF). Considering two samples $x$ and $x^{\prime}$ in form feature vectors, the mathematical representation of each kernel is presented here: (4) Linear, (5) Polynomial, (6) Sigmoid, (7) Radial Basis Function.

$$
\begin{aligned}
& K\left(x, x^{\prime}\right)=x^{T} x^{\prime} \\
& K\left(x, x^{\prime}\right)=\left(x^{T} x^{\prime}+c\right)^{d} \\
& K\left(x, x^{\prime}\right)=\tanh \left(a x^{T} x^{\prime}+r\right) \\
& K\left(x, x^{\prime}\right)=\exp \left(-\frac{\left\|x-x^{\prime}\right\|^{2}}{2 \sigma^{2}}\right)
\end{aligned}
$$

The hyper parameter space for performing Grid Search is as follows: $\quad C \in\left[10^{-10}, 10^{0}, 4\right], \varepsilon \in\left[10^{-8}, 10^{-1}, 4\right], d \in[3$ or 9$]$, coef $0 \in\left[10^{-10}, 10^{0}, 4\right], \gamma \in\left[10^{-20}, 10^{-1}, 4\right]$, where $C$ is the penalty parameter in the error term, $\varepsilon$ represents the tolerance radius of a prediction within which no loss is incurred, $d$ is the degree of the polynomial kernel, and coef 0 is an independent term used by polynomial and sigmoid kernels. The three-number notation $[l, u, n]$ denotes $n$ points spaced evenly on log scale in range $[l, u]$.

\section{MTGA WORKFLOW}

To demonstrate the effectiveness and generalization of our forecasting framework, we use the Microbiome Taxonomy and Gene Abundance workflow (MTGA) [32] as our use case. The workflow has been implemented using bioKepler [33]. As shown in Fig 5, the MTGA workflow consists of distinct tasks which are fairly independent in terms of the algorithms. It is both compute and data intensive. It gives us ability to test our framework's performance in new scenarios by asking the framework to predict on different parts of the MTGA workflow. The workflow also provides varied level of complexity in terms of potential for parallelization.

We are focused on four key workflow modules (colored yellow in Fig 5) for this task: CD-HIT-DUP (A), FR-HIT (B), Velvet-G (C), Velvet-H (D). Here onwards we will use the letters $A, B, C, D$ to refer to respective task.

CD-HIT-DUP (A) is a tool that removes duplicates from sequencing reads. FR-HIT (B) performs fragment recruitment, which 
aligns input sequence to a reference genome. Velvet-H (D) is a tool that performs hashing of the input and has information about meaning of input files. This feeds into Velvet-G, which builds a graph from the data obtained from Velvet-G (C). Velvet-G (C) performs further analysis such as simplification, error correction and extractions of processed information on this graph.

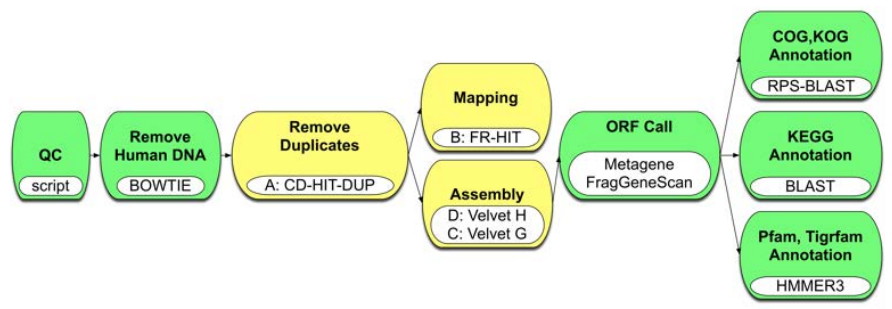

Fig 5 Representative use case: Microbiome Taxonomy and Gene Abundance workflow (MTGA). The MTGA workflow consists of distinct tasks which are fairly independent in terms of the algorithms. It is both compute and data intensive.

\section{STRICT TRAINING - DISJOINT SETS}

In the most general sense, there are $2^{4}-1$ ways of partitioning a profiled dataset with four distinct executables. These cases correspond to selecting samples from 1,2, 3, or all 4 executables respectively (i.e. ${ }^{4} \mathrm{C}_{1},{ }^{4} \mathrm{C}_{2},{ }^{4} \mathrm{C}_{3},{ }^{4} \mathrm{C}_{4}$ ). In the results reported here, we consider the scenario when 2 or 3 modules are selected for training. In each case the left out executables are used for testing a model's strength on prediction accuracy. Samples belonging to executables in the test set are strictly not allowed in the training set, hence the name Strict Training.

The strict training gives us the ability to test a model's performance in the context of workflows when there is lack of data about a new workflow being run on a machine. This scenario is common, hence getting information about models that can extract relevant characteristics from executables which allows them to scale to never-seen executables is valuable.

\section{Linear $\left({ }^{4} \mathrm{C}_{2}\right)$ :}

We explain here the notation used in plotting the RMSE scores of each experiment. This applies to each graph showing model performance on a test set. The $\mathrm{x}$-axis labels are of the form $X Y \rightrightarrows$ $P Q$. It signifies that the training set contains samples from $\mathrm{X} \& \mathrm{Y}$, and the test set contains samples from $P \& Q$. The results for each module in the test set are presented in ascending order and separated by an empty bar. For example, in the case of $A B \rightrightarrows C D$, the results for $C$ appears before those for D. For each module, first, the mean of observed execution time of all samples in the test set is plotted, followed by respective RMSE scores of the given model. We plot the observed execution times to enable comparision of RMSE with it. An RMSE of 100 seconds might be acceptable for a module with an observered mean execution time of 2000 seconds, but not acceptable for another module that takes 10 seconds to execute. The test modules for the first case are annotated. When more than one module is present in the test set, the modules are presented in ascending order. The negative number $(-100)$ signifies an extremely large RMSE score. One possible reason for this behavior could be algorithm's nonconvergence in that scenario.

Linear model with data from only two modules represents minimal data and simplest model scenario in our range of experiments. Fig 6 shows performance of linear models for $6\left({ }^{4} \mathrm{C}_{2}\right)$ possible ways of selecting two out of four modules for training. We use samples from remaining modules for testing.

The linear models Lasso and ElasticNet coincide in their performance and are plotted together. This occurs because ElasticNet essentially chooses an optimal point between Ridge and Lasso regularization penalties. In this case, the optimal performance comes from inclining towards Lasso. Considering cases when RMSE is less than $60 \%$ of the expected test set mean, the linear models perform well in some cases $(A B \rightrightarrows C, C D \rightrightarrows B, A C \rightrightarrows B D, B D \rightrightarrows C)$, which is non-trivial, since they are forecasting on modules that they have never learnt directly from. We note that error scores on module A suggest that it is hardest to forecast. The Grid Search performs worst for module $\mathrm{A}$ in all three models: Lasso, Ridge and ElasticNet.

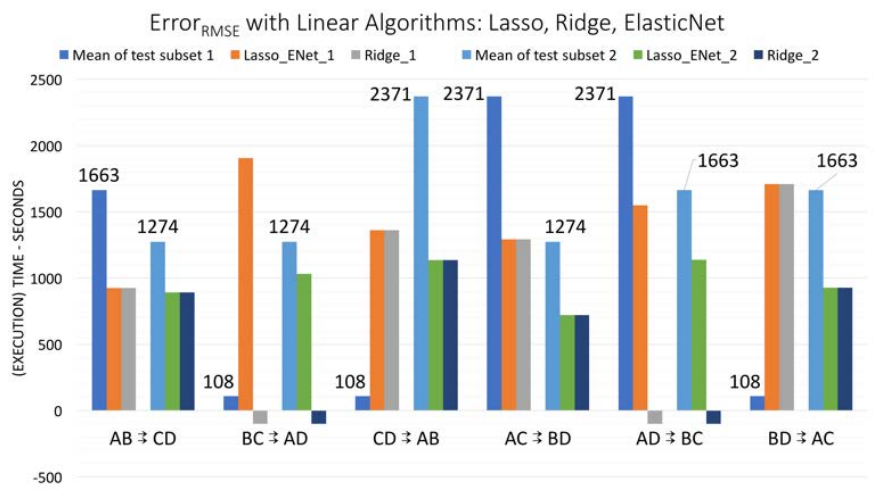

Fig 6 When Linear models learn from 2 modules and make predictions about remaining 2 modules, without training from a single sample from the new modules, we see sporadic performance with RMSE $<60 \%$ of observed mean in some cases. Note that Lasso and ElasticNet coincide in performance hence are represented together. $\mathrm{XY} \rightrightarrows \mathrm{PQ}$ means that module $\mathrm{P}$ is $1^{\text {st }}$ test subset, $\mathrm{Q}$ is the $2^{\text {nd }}$ test subset and the model is trained on $\mathrm{X}$ and $\mathrm{Y}$ modules.

\section{Linear $\left({ }^{4} C_{3}\right)$ :}

When more information is provided in the $4 \mathrm{C} 3$ training phase, all three linear models coincide in their performance. As shown in Fig 7, when compared to themselves with less data, Linear models do improve in most cases, however, we see slight decrease in accuracy for few cases. The RMSE on module A is drastically large compared to the observed mean execution time of $\mathrm{A}$. Linear models learn to predict well for module $\mathrm{B}$ and $\mathrm{C}$, from $\mathrm{CDA}$ and $\mathrm{DAB}$ respectively. This is worth noting since no sample leaked from $B$ or $C$ into the training phase. Using RMSE as precision metric, we see that Linear models can give reliable predictions for unseen workflow modules even when data is scarce.

\section{Ensemble $\left({ }^{4} \mathrm{C}_{2}\right)$ :}

When forced to learn from less amount of data, ensemble methods give encouraging results for several cases ( $A B \rightrightarrows$ $C D, A D \rightrightarrows C, B D \rightrightarrows C)$. Ensemble models rank much higher in complexity space when compared to linear learning models. Fig 6 and show that Linear models still manage to outperform Ensemble methods, when competing in data-scarce environment $(C D \rightrightarrows$ $B, A C \rightrightarrows B D, B D \rightrightarrows A)$. It is noteworthy that Ensemble methods also give high error values for module $A$, in the strict training scenario. When profiled data is in scarcity, linear models provide a viable alternative as a lightweight workflow module predictor. This suggests that a two-layer predictor that uses linear methods for new 
workflow modules, and more advanced methods for historically available modules can be a feasible direction to explore.

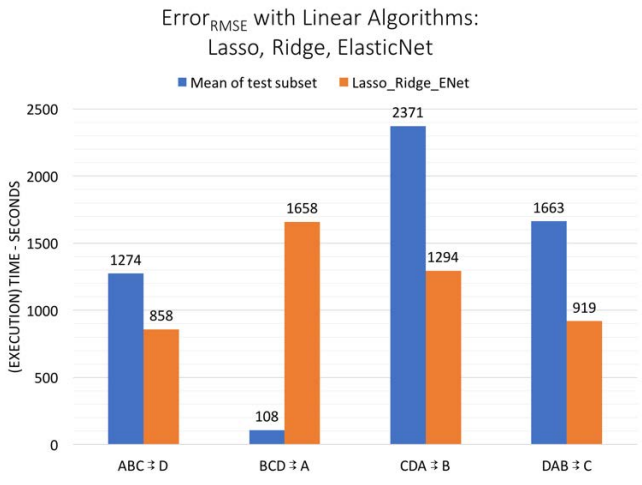

Fig 7 With extra information i.e. learning from 3 modules, Linear models still struggle to predict accurately on some modules. In this graph, linear models perform poorly on module A. XYZ $\rightarrow \mathrm{P}$ means that $\mathrm{P}$ is the test subset and the model is trained on $\mathrm{X}, \mathrm{Y}$ and $\mathrm{Z}$ modules.

\section{Ensemble $\left({ }^{4} \mathrm{C}_{3}\right)$ :}

Ensemble methods improve their performance for some cases, when provided with more information during strict training as shown in Fig 9. Gradient Boosting does well in $A B C \rightrightarrows D$ case. Both models perform well for $D A B \rightrightarrows C$ scenario. $B C D$ do not provide any help in predicting about $A$ for both Gradient Boosting and Random Forest. We note that even advanced models such as Ensemble based learning do not provide a viable solution across all modules under strict training scenarios, when no sample from test set can leak to the training set. This demonstrates the necessity of performance profiling for developing workflow performance predictors that can generate reliable estimates for schedulers.

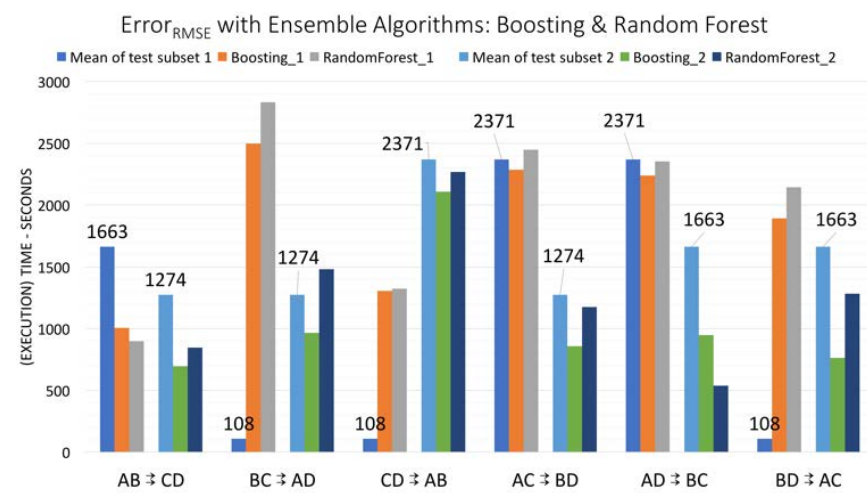

Fig 8 When Ensemble models learn from 2 modules and make predictions about remaining 2 modules, without training from a single sample from the new modules, Ensemble models outperform linear models in majority of the scenarios $(8 / 12)$. XY $\rightarrow \mathrm{PQ}$ means that module $\mathrm{P}$ is $1^{\text {st }}$ test subset, $\mathrm{Q}$ is the $2^{\text {nd }}$ test subset and the model is trained on $\mathrm{X}$ and $\mathrm{Y}$ modules.

\section{Support Vector Machines $\left({ }^{4} \mathrm{C}_{2}\right)$ :}

Support Vector Machines are widely accepted for their high predictive performance, hence we included SVM regression in our tests to evaluate them against workflow modules. Negative values in Fig 10 signify that SVM gives high error values when under the stress of data scarcity. Sigmoid, Linear and RBF kernels coincide in their performance after the parametric Grid Search, and polynomial kernel evaluates to different outcomes. During extreme case of strict training, the SVM regression performs well under some scenarios: $A B \rightrightarrows C, C D \rightrightarrows B, A C \rightrightarrows D, B D \rightrightarrows C$. Linear models give a tough competition to SVM models under scarce data conditions. They outperform in many cases: $A B \rightrightarrows C D, C D \rightrightarrows B, A C \rightrightarrows B D, B D \rightrightarrows A$. Linear models might be a valuable resource to consider when developing predictors for data scarce scenarios.

\section{Support Vector Machines $\left({ }^{4} \mathrm{C}_{3}\right)$ :}

When compared with itself under less training data, Support Vector Machine based regression models improve in some cases as shown in Fig 11. SVM regression gives tolerable performance against other models when given more data under strict training. Linear and Ensemble methods outperform SVM kernels in 3 out of 4 cases $(A B C \rightrightarrows D, B C D \rightrightarrows A, C D A \rightrightarrows B)$. Overall, none of the SVM kernels give reliable forecasts for all scenarios under strict training. Using RMSE as error measure, Linear models come out as surprising contenders in data-scarce scenarios.

\section{Error $_{\text {RMSE }}$ with Ensemble Algorithms: Gradient Boosting, Random Forest}

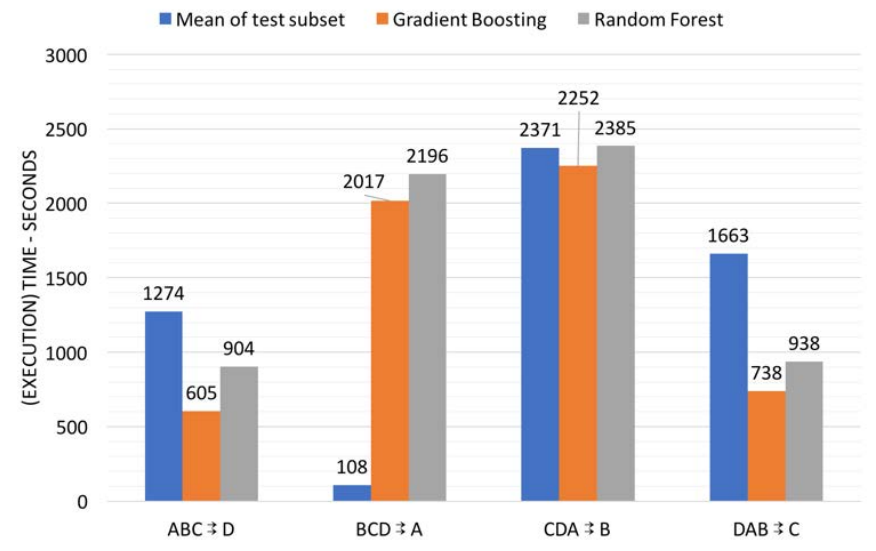

Fig 9 With extra information i.e. learning from 3 modules, Ensemble models still struggle to predict accurately on some modules. Surprisingly, Linear model outperform Ensemble models in 2 (A and B) out of 4 cases

\section{LENIENT TRAINING - INTERSECTING SETS}

The idea behind lenient training is that if a model has already seen how a given executable performs on a particular resource, it is likely to predict well for a different resource and input data size with regards to the same executable. In contrast, the predictor is in a tough situation when it has never seen a single instance of how a given executable performs on any resource and input combination. We analyze the use of lenient learning to tackle the cold start problem of predicting a workflows performance when some modules of the workflows were never executed before. In the lenient training, we allow the model to witness and learn from few samples from executables that are also present in test set. However, we do not give it any sample directly from the test set. It is always different from every sample of the same executable present in the test set. 


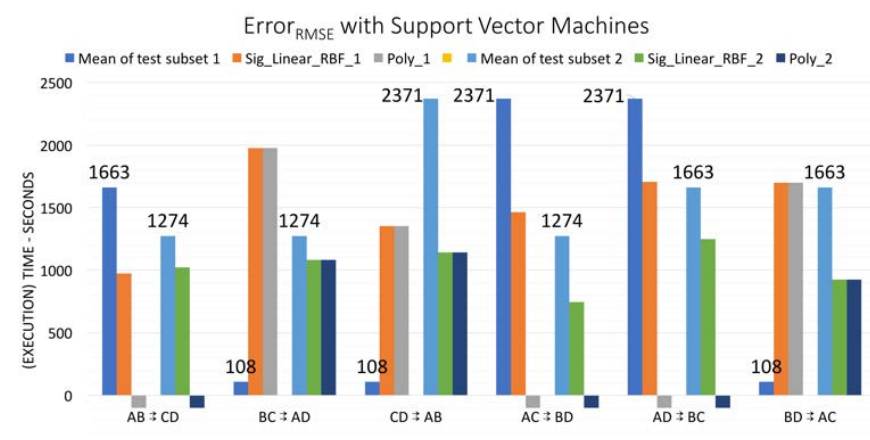

$-500$

Fig 10 When SVM models learn from 2 modules and make predictions about remaining 2 modules, without training from a single sample from the new modules, SVM models outperform linear models in half of the scenarios (6/12). XY $\rightrightarrows \mathrm{PQ}$ means that module $\mathrm{P}$ is $1^{\text {st }}$ test subset, $\mathrm{Q}$ is the $2^{\text {nd }}$ test subset and the model is trained on $\mathrm{X}$ and $\mathrm{Y}$ modules. Sigmoid, Linear and RBF kernels coincide in performance and are represented together.

The lenient training corresponds to the ${ }^{4} \mathrm{C}_{4}$ partition case, since all executables have found their way to the training set. We present the results for three class of Machine Learning algorithms: Linear models, Ensemble methods, and Support Vector Machines (SVM) models. We will use the performance of the Linear Models as our baseline for comparison with other models.

When a model is given samples from an executable, it learns about the executable's behavior in a specific setting. A model that can extract enough information to generalize to 'any' coupling of that executable with another resource setting and different input data size, is representative of best case scenario. In the results presented in this section, we see how well ensemble and SVM perform in comparison to linear models, when given more information.

\section{Linear $\left({ }^{4} \mathrm{C}_{4}\right)$ :}

Linear models gain performance in every case when compared to themselves with less data in the strict training phase. Fig 12 shows that RMSE performance of Linear models Lasso and ElasticNet coincide during the lenient training. In lenient training, samples from all modules are available in the training session. The error decreases significantly when compared to the strict training case $\left({ }^{4} \mathrm{C}_{2}\right.$ and $\left.{ }^{4} \mathrm{C}_{3}\right)$. Strict training occurs when a predictor is forced to forecast module performance without looking at a single sample from that module. In three cases, Linear models give RMSE score that is less than $60 \%$ of the expected mean on the unseen test set: $A B C D \rightrightarrows B, A B C D \rightrightarrows$ $C, A B C D \rightrightarrows D$.

\section{Ensemble $\left({ }^{4} \mathrm{C}_{4}\right)$ :}

The Ensemble models flourish when given access to samples from each module in the test set. The performance improves drastically when compared to their own behavior in the data-scarce strict training scenarios, as shown in Fig 13. The superior performance on module A is worth noting, especially because every model struggled to forecast A when it was restricted access during the strict training. The ensemble methods outperform themselves when compared to every strict training scenario they were stress tested in. At least one of the Ensemble methods is able to outperform Linear models in every lenient training case, when given access to samples from all modules. This demonstrates that Ensemble methods are likely to perform better than linear models, when there are plenty of training data points.

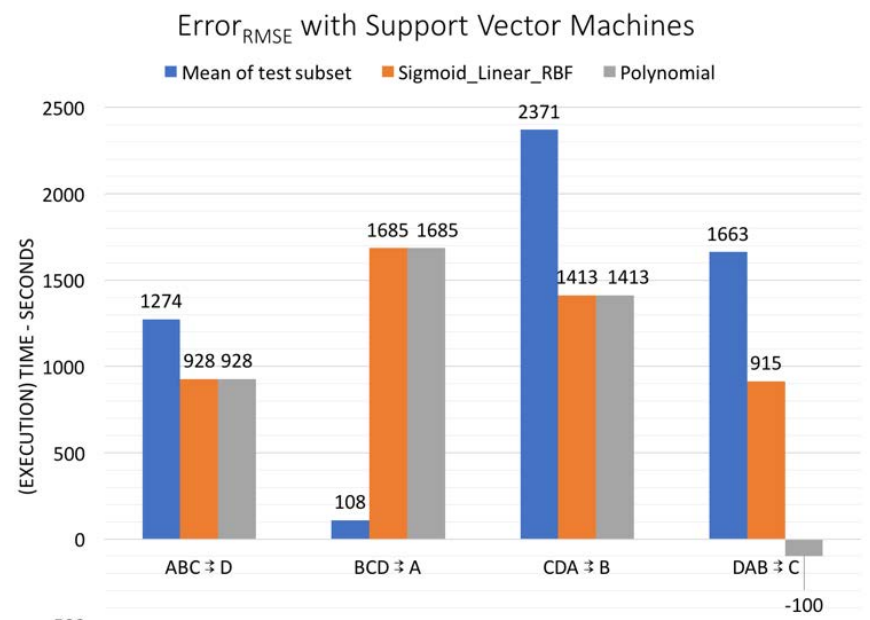

Fig 11 With extra information i.e. learning from 3 modules, SVM models still struggle to predict accurately on some modules. Surprisingly, Linear model outperform Ensemble models in 3 (A, B and $\mathrm{D}$ ) out of 4 cases. Under data starvation, Linear models can give results that are comparable or better than complex models.

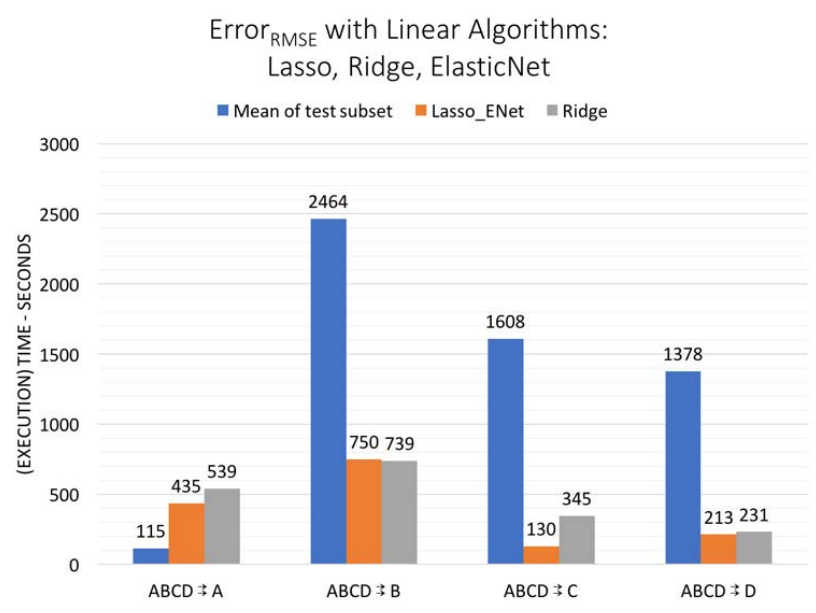

Fig 12 When Linear models get samples from all modules, they perform excellent on some modules $(\mathrm{C}, \mathrm{D})$, but do not give reliable results for all modules.

\section{Support Vector Machines $\left({ }^{4} \mathrm{C}_{4}\right)$ :}

SVM regression gain performance in most cases as more data is provided. Fig 14 shows that SVM gains significant performance improvement with Polynomial Kernel for modules A and D. In fact, it even outperforms Ensemble methods for modules D under lenient training. However, Linear models outperform SVM for two cases 
$(A B C D \rightrightarrows B, A B C D \rightrightarrows C$ ), showing that it is not an overall reliable model for all modules in lenient training.

We note the exceptional performance of Polynomial kernel for two distinct workflow modules here. SVM models require heavy computation when compared to Linear and Ensemble based methods, and rank lowest in several lenient training cases. Performance of SVM kernels varies across different domains, and choosing which kernel to use for a specific problem is a non-trivial task. More experiments are needed to make a larger claim about Polynomial kernel's affinity to predict workflows.

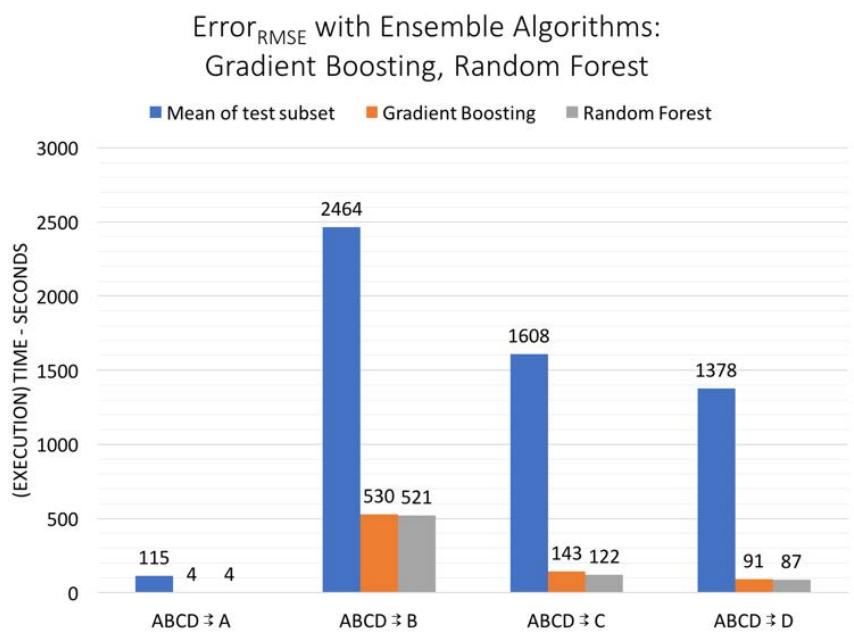

Fig 13 Ensemble methods give outstanding performance across all modules, when given chance to learn about each module. In this graph, we see drastic improvement on module A (low error), a module on which all models struggled when under data deprivation.

Overall comparison of all Machine Learning models tested shows that Ensemble Methods consistently perform well for lenient prediction of workflow modules as shown in Fig.15.

\section{FEATURE SET REDUCTION}

Features capture characteristics of a system in discrete form by measuring an event's contribution towards a given feature. More number of features help the learning model find fine-tuned information about an event we want to predict. However, the requirement for training data grows exponentially with respect to features. In case of less data, we want to reduce the number of features prudently, to capture enough information with currently available data. For example, it might make sense to drop a feature which increases prediction accuracy by least amount among all the features.

In a quest to reduce complexity and data requirement, we aim to find a compact representation of a module's workload and hardware fingerprint, that uses minimal attributes. To find the optimal number of features, we perform feature set reduction using recursive removal of features and cross-validated filtering [34]

In this strategy, we start with a model that is initially trained using all the features. The model prioritizes each feature based on its utility in the prediction process. A smaller subset of initial feature set is generated by eliminating the featured with low priorities. This process is recursively carried out until a base case characterizing the expected 'optimality' is reached.

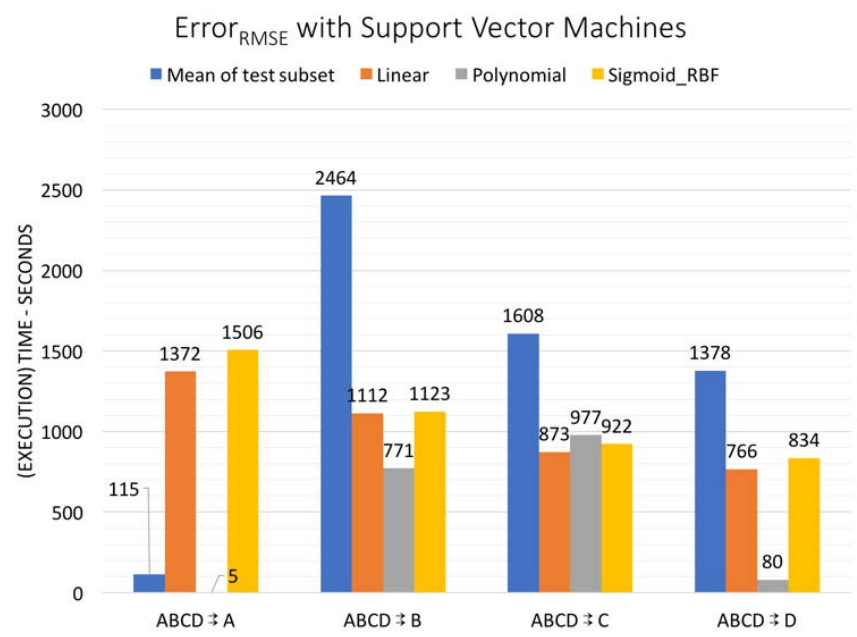

Fig 14 Support Vector Machine based models perform sporadically even when given training samples from all modules. Polynomial Kernel shows fairly good performance. Linear still outperforms in 2 (B, C) / 4 cases. Support Vector Machines can give precise results for some modules (here A, D).

Error $_{\text {RMSE }}$ with Ensemble Algorithms: Gradient Boosting, Random Forest

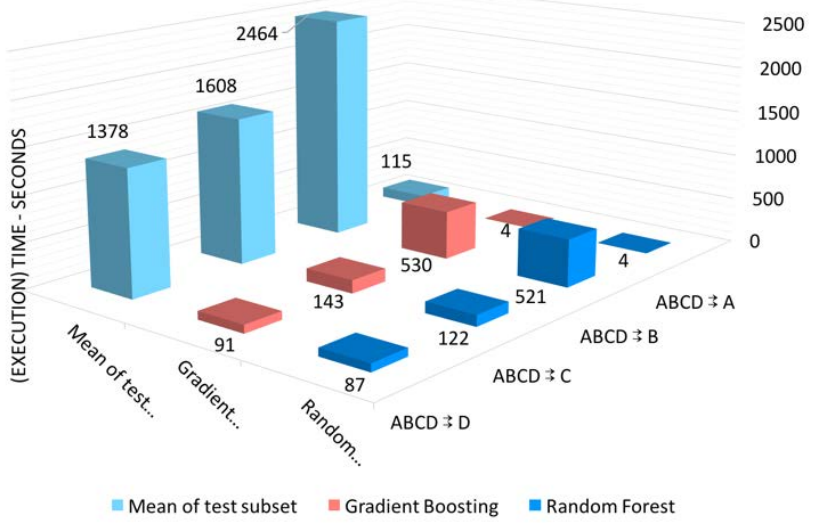

Fig 15 Ensemble methods show outstanding performance across different modules, when given training samples from all modules. Ensemble methods could be candidate for real time learning, and leading to improvements in prediction as the models gain more experience from new workflows.

The final number of features is chosen using the average performance score across all folds of the cross-validation process. The number of features that results in highest model score considering all folds is chosen to be the optimal number.

Using the Random Forest Regressor, a before and after comparison of the RMSE score under the lenient training to measure the effect of feature set reduction performed. The feature reduction is performed using a 3 fold cross validation strategy. The algorithm results in selecting 8 out of 57 features reducing 1 feature during each step. The features which filter out as most significant for 
predicting execution time are [size of input, 'ft4', 'ft39', 'ft34', 'ft37', 'ft47', 'ft51', 'ft29'] as shown in Table I.

\section{TABLE I. 8 out of 57 features that ranked highest in significance to predict execution time of modules}

\begin{tabular}{|c|c|}
\hline Feature & Significance \\
\hline 'sizeGB' & Dataset size used as input \\
\hline 'ft4' & \# of basic blocks with more than two successors \\
\hline ' ft29' & \# of basic blocks with phi nodes in the interval $[0,3]$ \\
\hline ' ft34' & \# of unary operations \\
\hline ' $\mathrm{ft} 37$ ' & \# of times the address of a variables is taken ("\\
&" in C) \\
\hline 'ft39' & \# of indirect calls (i.e. done via pointers) \\
\hline ' ft47' & \# of occurrences of 32-bit integer constants \\
\hline ' ft51' & \# of references (def/use) of static/extern variables \\
\hline
\end{tabular}

We use the fine-tuned model generated by RFECV (Recursive Feature Elimination coupled with Cross-Validated filtering) to predict on workflow sub-modules $\mathrm{A}, \mathrm{B}, \mathrm{C}$ and $\mathrm{D}$ using the reduced feature sets. Fig 16 shows comparison of performance between two cases: when random forest regressor is exposed to all 57 features and when it has limited access to only 8 features. It is noteworthy that the performance of Random Forest improves in all four cases, when using reduced feature set.

The reason for improvement of performance is inherent in the Random Forest Regressor: it picks a feature randomly to split nodes. When it has access to limited but highly relevant features, algorithm picks an impactful feature for splitting in all cases, resulting in improvement in comparison to a large set of features with significant variation in their relevance to the prediction process. This experiment leads us to noteworthy conclusion that Random Forest is expected to perform better with fewer features, if they are assured to be substantial in the process of forecasting.

\section{CONCLUSION \& FUTURE WORK}

We present a framework that breaks the task of forecasting a complex workflow's performance footprint into many smaller tasks that are bounded by physical limits of the resource where they execute. We create an abstraction called 'module' which unites an executable and the resource it runs on, as a single unit of prediction. The unification makes the workflow forecasting problem tractable, and coagulates factors that can significantly impact performance of code when executed on a machine. This modular approach leaves room for improving individual module level forecasting techniques, and has place for accepting future predictors that will specialize in forecasting ever growing set of workflows and hardware platforms.

The results presented in this paper give researchers an insight into selecting optimal Machine Learning models for their workflow performance forecasting needs. Model performance varies as training data set size changes, and the resource allocation changes. This article shows which models are suitable for scarce data scenarios, which can be useful for researchers venturing into predicting newly developed workflows. We discover that Ensemble methods perform well for mature workflows for which there is enough data to leak out few samples for the training phase. This paper acts as a proof of concept of the idea. In future, we would like to build the proposed underlying framework and incorporate it with the Kepler workflow system's provenance module, thus enhancing scheduling capabilities of large scale workflows.

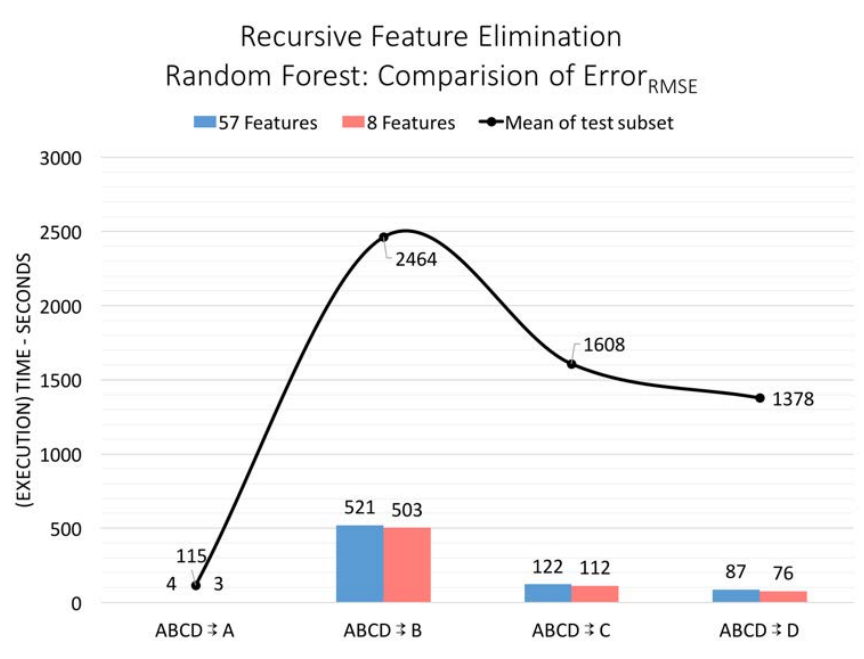

Fig 16 Comparison of performance between two cases: Random Forest regressor with all 57 features and when it has limited access to only 8 features. Recursive Feature Elimination with Cross Validation resulted in a 7 fold reduction of features, which preserving the model's prediction capability.

\section{Acknowledgment}

This work is supported by NSF DBI 1062565 and 1331615, NIH P41 GM103426 for NBCR, and DOE DE-SC0012630 for IPPD. The content is solely the responsibility of the authors and does not necessarily represent the official views of the funding agencies.

\section{References}

[1] R. Gibbons, "A historical application profiler for use by parallel schedulers," in fob Scheduling Strategies for Parallel Processing, 1997, pp. 58-77.

[2] E. Deelman et al., "The future of scientific workflows," Int. F. High Perform. Comput. Appl., p. 1094342017704893, Apr. 2017.

[3] E. Deelman, D. Gannon, M. Shields, and I. Taylor, "Workflows and eScience: An Overview of Workflow System Features and Capabilities," Future Gener Comput Syst, vol. 25, no. 5, pp. 528-540, May 2009.

[4] V. Taylor, X. Wu, and R. Stevens, "Prophesy: An Infrastructure for Performance Analysis and Modeling of Parallel and Grid Applications," SIGMETRICS Perform Eval Rev, vol. 30, no. 4, pp. 1318, Mar. 2003.

[5] H. Hiden, S. Woodman, and P. Watson, "A Framework for Dynamically Generating Predictive Models of Workflow Execution," in Proceedings of the 8th Workshop on Workflows in Support of LargeScale Science, New York, NY, USA, 2013, pp. 77-87.

[6] K. Hoste, A. Phansalkar, L. Eeckhout, A. Georges, L. K. John, and K. De Bosschere, "Performance Prediction Based on Inherent Program Similarity," in Proceedings of the 15th International Conference on Parallel Architectures and Compilation Techniques, New York, NY, USA, 2006, pp. 114-122.

[7] E. Ïpek, S. A. McKee, R. Caruana, B. R. de Supinski, and M. Schulz, "Efficiently Exploring Architectural Design Spaces via Predictive Modeling," in Proceedings of the 12th International Conference on Architectural Support for Programming Languages and Operating Systems, New York, NY, USA, 2006, pp. 195-206.

[8] B. C. Lee and D. M. Brooks, "Accurate and Efficient Regression Modeling for Microarchitectural Performance and Power Prediction," in Proceedings of the 12th International Conference on Architectural 
Support for Programming Languages and Operating Systems, New York, NY, USA, 2006, pp. 185-194.

[9] F. Nadeem and T. Fahringer, "Predicting the Execution Time of Grid Workflow Applications Through Local Learning," in Proceedings of the Conference on High Performance Computing Networking, Storage and Analysis, New York, NY, USA, 2009, p. 33:1-33:12.

[10] I. Pietri, G. Juve, E. Deelman, and R. Sakellariou, "A Performance Model to Estimate Execution Time of Scientific Workflows on the Cloud," 2014, pp. 11-19.

[11] A. Matsunaga and J. A. B. Fortes, "On the Use of Machine Learning to Predict the Time and Resources Consumed by Applications," in 2010 10th IEEE/ACM International Conference on Cluster, Cloud and Grid Computing, 2010, pp. 495-504.

[12] F. Nadeem and T. Fahringer, "Using Templates to Predict Execution Time of Scientific Workflow Applications in the Grid," in 2009 9th IEEE/ACM International Symposium on Cluster Computing and the Grid, 2009, pp. 316-323.

[13] T. Miu and P. Missier, "Predicting the Execution Time of Workflow Activities Based on Their Input Features," in Proceedings of the 2012 SC Companion: High Performance Computing, Networking Storage and Analysis, Washington, DC, USA, 2012, pp. 64-72.

[14] P. Shivam, S. Babu, and J. Chase, "Active and Accelerated Learning of Cost Models for Optimizing Scientific Applications," in Proceedings of the 32Nd International Conference on Very Large Data Bases, Seoul, Korea, 2006, pp. 535-546.

[15] A. A. Bankole and S. A. Ajila, "Predicting cloud resource provisioning using machine learning techniques," in 2013 26th IEEE Canadian Conference on Electrical and Computer Engineering (CCECE), 2013, pp. 1-4.

[16] S. Kundu, R. Rangaswami, A. Gulati, M. Zhao, and K. Dutta, "Modeling Virtualized Applications Using Machine Learning Techniques," in Proceedings of the 8th ACM SIGPLAN/SIGOPS Conference on Virtual Execution Environments, New York, NY, USA, 2012, pp. 3-14.

[17] C. Stewart, T. Kelly, A. Zhang, and K. Shen, "A Dollar from 15 Cents: Cross-platform Management for Internet Services," in USENIX 2008 Annual Technical Conference, Berkeley, CA, USA, 2008, pp. 199-212.

[18] R. H. Saavedra and A. J. Smith, "Analysis of Benchmark Characteristics and Benchmark Performance Prediction," ACM Trans Comput Syst, vol. 14, no. 4, pp. 344-384, Nov. 1996.

[19] R. F. da Silva et al., "Toward Fine-grained Online Task Characteristics Estimation in Scientific Workflows," in Proceedings of the 8th Workshop on Workflows in Support of Large-Scale Science, New York, NY, USA, 2013, pp. 58-67.

[20] M. Dobber, R. van der Mei, and G. Koole, "Effective Prediction of Job Processing Times in a Large-Scale Grid Environment," in 2006 15th
IEEE International Conference on High Performance Distributed Computing, 2006, pp. 359-360.

[21] T. Glatard, J. Montagnat, and X. Pennec, "A Probabilistic Model to Analyse Workflow Performance on Production Grids," in Proceedings of the 2008 Eighth IEEE International Symposium on Cluster Computing and the Grid, Washington, DC, USA, 2008, pp. 510-517.

[22] M. Tao, S. Dong, and L. Zhang, "A multi-strategy collaborative prediction model for the runtime of online tasks in computing cluster/grid," Clust. Comput., vol. 14, no. 2, pp. 199-210, Jun. 2011.

[23] X. Liu et al., "A Novel Statistical Time-series Pattern Based Interval Forecasting Strategy for Activity Durations in Workflow Systems," 7 Syst Softw, vol. 84, no. 3, pp. 354-376, Mar. 2011.

[24] B. Ludäscher et al., "Scientific Workflow Management and the Kepler System: Research Articles," Concurr Comput Pr. Exper, vol. 18, no. 10, pp. 1039-1065, Aug. 2006.

[25] G. Fursin et al., "Milepost GCC: Machine Learning Enabled Selftuning Compiler," Int. f. Parallel Program., vol. 39, no. 3, pp. 296-327, Jun. 2011.

[26] "CTools:MilepostGCC:StaticFeatures:MILEPOST V2.1 - cTuning.org." [Online]. Available: http://ctuning.org/wiki/index.php/CTools:MilepostGCC:StaticFeatur es:MILEPOST_V2.1. [Accessed: 13-Aug-2017].

[27] F. Pedregosa et al., "Scikit-learn: Machine Learning in Python," $f$ Mach Learn Res, vol. 12, pp. 2825-2830, Nov. 2011.

[28] L. Breiman, "Random Forests," Mach. Learn., vol. 45, no. 1, pp. 5-32, Oct. 2001.

[29] J. H. Friedman, "Greedy Function Approximation: A Gradient Boosting Machine,” Ann. Stat., vol. 29, pp. 1189-1232, 2000.

[30] C. Cortes and V. Vapnik, "Support-vector networks," Mach. Learn., vol. 20, no. 3, pp. 273-297, Sep. 1995.

[31] A. J. Smola and B. Schölkopf, A tutorial on support vector regression. 2004.

[32] S. Wu, W. Li, L. Smarr, K. Nelson, S. Yooseph, and M. Torralba, "Large Memory High Performance Computing Enables Comparison Across Human Gut Microbiome of Patients with Autoimmune Diseases and Healthy Subjects," in Proceedings of the Conference on Extreme Science and Engineering Discovery Environment: Gateway to Discovery, New York, NY, USA, 2013, p. 25:1-25:6.

[33] I. Altintas, J. Wang, D. Crawl, and W. Li, "Challenges and Approaches for Distributed Workflow-driven Analysis of Large-scale Biological Data: Vision Paper," in Proceedings of the 2012 foint EDBT/ICDT Workshops, New York, NY, USA, 2012, pp. 73-78.

[34] I. Guyon, J. Weston, S. Barnhill, and V. Vapnik, "Gene Selection for Cancer Classification using Support Vector Machines," Mach. Learn., vol. 46, no. 1-3, pp. 389-422, Jan. 2002. 